\title{
An African Tick Flavivirus Forming an Independent Clade Exhibits Unique Exoribonuclease-Resistant RNA Structures in the Genomic 3'-Untranslated Region
}

\author{
Hayato Harima \\ Hokkaido University \\ Yasuko Orba \\ Hokkaido University \\ Shiho Torii \\ Hokkaido University

\begin{abstract}
Yongjin Qiu
Hokkaido University

Masahiro Kajihara

Hokkaido University

Yoshiki Eto

Hokkaido University
\end{abstract} \\ Naoya Matsuta \\ Niigata University \\ Bernard Hang'ombe \\ University of Zambia \\ Yuki Eshita \\ Hokkaido University \\ Kentaro Uemura \\ Hokkaido University \\ Keita Matsuno \\ Hokkaido University \\ Michihito Sasaki \\ Hokkaido University \\ Kentaro Yoshii \\ Nagasaki University \\ Ryo Nakao \\ Hokkaido University \\ William Hall \\ University College Dublin

\section{Ayato Takada} \\ Hokkaido University \\ Takashi Abe \\ Niigata University \\ Michael Wolfinger
}


University of Vienna

\section{Martin Simmunza}

University of Zambia

Hirofumi Sawa ( $\sim$ h-sawa@czc.hokudai.ac.jp)

Hokkaido University

\section{Research Article}

Keywords: TBFVs, MPFV, Flavivirus

Posted Date: December 1st, 2020

DOI: https://doi.org/10.21203/rs.3.rs-107044/v1

License: (c) (i) This work is licensed under a Creative Commons Attribution 4.0 International License. Read Full License

Version of Record: A version of this preprint was published at Scientific Reports on March 1st, 2021. See the published version at https://doi.org/10.1038/s41598-021-84365-9. 


\section{Abstract}

Tick-borne flaviviruses (TBFVs) infect mammalian hosts through tick bites and can cause various serious illnesses, such as encephalitis and hemorrhagic fevers, both in humans and animals. Despite their importance to public health, there is limited epidemiological information on TBFV infection in Africa is limited. Herein, we report that a novel flavivirus, Mpulungu flavivirus (MPFV), was discovered in a Rhipicephalus muhsamaetick in Zambia. MPFV was found to be genetically related to Ngoye virus detected in ticks in Senegal, and these viruses formed a unique lineage in the genus Flavivirus. Analyses of dinucleotide contents of flaviviruses indicated that MPFV was similar to those of other TBFVs with a typical vertebrate genome signature, suggesting that MPFV may infect vertebrate hosts leading to significant public health problems. Bioinformatic analyses of the secondary structures in the 3'untranslated regions (UTRs) revealed that MPFV exhibits unique exoribonuclease-resistant RNA (xrRNA) structures. Utilizing biochemical approaches, we clarified that two xrRNA structures of MPFV in the 3'-UTR could prevent exoribonuclease activity. In summary, our findings provide new information regarding the geographical distribution of TBFV and xrRNA structures in the 3'-UTR of flaviviruses.

\section{Introduction}

The genus Flavivirus in the family Flaviviridae comprises more than 50 species, which can be divided into vectorborne flaviviruses, insect-specific flaviviruses (ISFVs), and no known arthropod vector flaviviruses (NKVs) ${ }^{1}$. Vectorborne flaviviruses are transmitted to vertebrate hosts by arthropod vectors, including ticks and mosquitos; a number of tick-borne flaviviruses (TBFVs) and mosquito-borne flaviviruses (MBFVs) cause serious illnesses, including encephalitis and hemorrhagic fevers, in humans and animals ${ }^{1,2}$. Flaviviruses associated with infectious diseases are distributed worldwide, and many people are facing the risk of TBFV and MBFV infections ${ }^{2}$.

To date, twelve TBFV species have been classified by the International Committee on Taxonomy of Viruses, and are classified into three groups: mammalian-TBFV group (Tick-borne encephalitis virus, Louping ill virus, Powassan virus, Kyasanur Forest disease virus, Langat virus, Omsk hemorrhagic fever virus, Gadgets Gully virus, and Royal Farm virus), seabird-TBFV group (Meaban virus, Saumarez Reef virus, and Tyuleniy virus), and probably-TBFV group

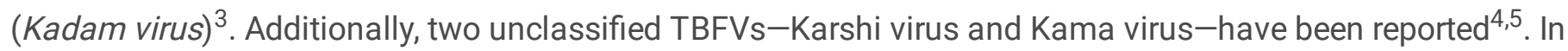
relation to clinical diseases, Alkhurma hemorrhagic fever viruses, a subtype of Kyasanur Forest disease virus, were identified in humans in Saudi Arabia in $1995^{6}$, and have subsequently detected in Egypt and Djibouti ${ }^{7-9}$. Kadam viruses classified as probable member of the TBFV have been isolated from ticks, including Rhipicephalus spp. and Amblyomma sp., in Uganda and Kenya ${ }^{10,11}$; however, it remains unknown whether these viruses are related to human and/or animal diseases. In recent years, genome fragments of a highly divergent flavivirus, Ngoye virus, were detected in Rhipicephalus evertsi evertsi and Rhipicephalus guilhoni in Senegal, indicating that tick-related flaviviruses exhibit large heterogeneity ${ }^{12}$. However, there is no information on the pathogenicity and prevalence of Ngoye virus in Africa. Therefore, we set out to screen flaviviruses in ticks, including Rhipicephalus spp., in Zambia.

Flaviviruses are enveloped, positive-sense single-stranded RNA viruses with a genome size of approximately 9-11 $\mathrm{kb}$, consisting of a 5'-untranslated region (UTR), single open reading frame (ORF), and a $3^{\prime}-\mathrm{UTR}^{1}$. The ORF encodes a single polyprotein composed of three structural proteins, namely the capsid (C), pre-membrane/membrane (prM) and envelop (E) proteins, and seven non-structural (NS) proteins: NS1, NS2A, NS2B, NS3, NS4A, NS4B, and NS5 ${ }^{1}$. These viral proteins are cleaved and matured from polyproteins by viral and host proteases ${ }^{13,14}$. The $5^{\prime}-$ UTR of flaviviruses is $\sim 100$ nucleotides in length, and the $3^{\prime}$-UTR is longer than $5^{\prime}$-UTR (400-700 nucleotides) ${ }^{15}$. 
Characteristic secondary structures of both the 5'-UTR and the 3'-UTR are required for genome cyclization, and interaction of the UTRs is essential for viral replication and translation ${ }^{16-22}$. During flavivirus infection, shorter subgenomic flavivirus RNAs (sfRNAs) are produced in addition to viral genomic RNAs ${ }^{23-28}$. The production of sfRNA is common to all flaviviruses, and is associated with viral pathogenicity, host adaptation, and immune evasion ${ }^{26-29}$. These sfRNAs originate from the 3'-UTR of the flavivirus genome and result from partial degradation of viral genomic RNA by the cellular 5'-3' exoribonuclease Xrn1, which degrades 5'-monophosphorylated RNAs in the cytoplasm ${ }^{23}$. Mechanistically, sfRNAs are produced by blocking the progression of Xrn1 from the $5^{\prime}$ end at specifically structured RNA elements in the 3'-UTR, which are termed exoribonuclease-resistant RNAs (xrRNAs). Interestingly, the secondary structures of TBFV xrRNAs differ from those of MBFV xrRNAs ${ }^{24}$. ISFV xrRNAs are conserved among the ISFV group and diverge from MBFV xrRNAs ${ }^{30}$, suggesting that classification of the flavivirus group according to these structural features may be of value. Although MBFV xrRNAs are relatively wellcharacterized, limited experimental data is available on the xrRNAs of other flaviviruses, including TBFV, ISFV, and $\mathrm{NKV}^{24}$. Therefore, functional characterization of secondary structures in 3'-UTRs is necessary to understand both the evolutionary history of flavivirus groups and viral adaptation to both arthropod vectors and vertebrate hosts.

Continued surveillance of TBFVs will provide key insights into the evolution of flaviviruses as well as vector/host relationship and adaptation. In this study, we attempted to detect TBFVs in field-collected ticks in Zambia. Herein, we describe the characterization of a novel flavivirus called Mpulungu flavivirus (MPFV), which was discovered in a Rhipicephalus muhsamae tick in Zambia and appears to be closely related to Ngoye virus. Complete genome sequencing, including the 5'- and 3'-UTRs, was conducted and analyzed for predicting host range. Utilizing biochemical approaches, we clarified that unique xrRNA structures in the 3'-UTR play a role in prevention of exoribonuclease digestion.

\section{Results}

\section{Discovery of a novel flavivirus in a Rhipicephalus muhsamae in Zambia}

To identify potential tick-borne pathogens in Zambia, ticks were collected from different areas in Zambia (i.e., Isoka, Mpulungu, and Samfya). To specifically examine flaviviruses in ticks, we screened a total of 573 individual ticks for detection of flaviviruses by reverse transcription-PCR (RT-PCR) using pan-flavivirus primers (see Supplementary Table S1 and S2 online). Of these, a single flavivirus RNA genome was detected from Rhipicephalus muhsamae collected in a pasture in Mpulungu; the resultant RT-PCR product ( $270 \mathrm{bp}$ ) was sequenced and analyzed using BLAST query (https://blast.ncbi.nlm.nih.gov/Blast.cgi). Sequencing demonstrated an $83.2 \%$ nucleotide identity with the Ngoye virus detected from Rhipicephalus evertsi evertsi and Rhipicephalus guilhoni in Senegal ${ }^{12}$. We attempted to isolate detected flavivirus from tick homogenates using intracerebral injections into neonatal mice and various cell lines, including African green monkey kidney (Vero E6), baby hamster kidney (BHK-21), Ixodes scapularis (ISE6), and Aedes albopictus (C6/36) cells. Several blind passages in neonatal murine brain and the aforementioned cell lines were performed; however, viral replication was not detected in either the brains of inoculated mice and passaged cell lines.

Viral genomic sequences contained in flavivirus-positive tick lysates were examined with next generation sequencing. A consensus flavivirus genome (10,596 nucleotides) was determined via bioinformatic analyses of the obtained nucleotide sequences. Based on the consensus sequence, rapid amplification of cDNA ends (RACE) was performed to determine the 5 '- and the 3 '- terminal sequences. The viral genome derived from Rhipicephalus muhsamae tick lysate was 10,868 nucleotides in length with a GC content of $56.5 \%$. BLAST analysis indicated that 
this detected flavivirus appeared to be most closely related to Ngoye virus with $83.7 \%$ nucleotide identity, even though the genomic sequence of Ngoye virus was only partially available in National Center for Biotechnology Information (NCBI) databank (4,176 nucleotides). This newly detected flavivirus was tentatively designated as Mpulungu flavivirus (MPFV). The determined genomic sequence of MPFV was deposited in the DNA Data Bank of Japan (DDBJ) under the accession number LC582740.

\section{MPFV forms an independent group among flaviviruses}

The predicted complete ORF sequence of MPFV was 10,206 nucleotides encoding a 3,401 amino acid polyprotein, and the 5'- and 3'-UTRs were 136 and 526 nucleotides in length, respectively (Table 1). The cleavage sites of both MPFV and representative flaviviruses were deduced (see Supplementary Table S3 online), and an identity comparison analysis based on amino acid sequence of each viral protein was conducted (Table 2). Identities based on the complete polyprotein sequences between MPFV and other representative flaviviruses-including TBFVs, MBFVs, ISFVs, and NKVs-were less than $45.0 \%$. Similar results were obtained by comparative analyses based on NS3, NS4A, NS4B, and NS5 proteins. Other MPFV non-structural proteins (NS1, NS2A, NS2B, and NS3) and the structural proteins (C, PrM, and E) were also highly divergent from known flaviviruses. However, the partial polyprotein of MPFV shared a high sequence identity (95.0\%) with Ngoye virus (Table 2).

NS3 and NS5 are multifunctional proteins in flaviviruses encoding enzymes required for polyprotein processing and RNA replication ${ }^{31-36}$. Serine protease and helicase/nucleoside-triphosphatase (NTPase) are encoded within the Nand C-terminal regions of NS3, respectively ${ }^{31-33}$, and NS5 contains a methyltransferase motif in the N-terminal domain as well as an RNA-dependent RNA polymerase (RdRp) in the C-terminal domain ${ }^{34-36}$. To analyze sequence conservation within these enzymatic motifs of NS3 and NS5, alignments were computed using MPFV sequences and those of other representative flaviviruses. The catalytic triad for serine protease activity, consisting of histidine, aspartic acid, and serine residues ${ }^{31}$, was conserved in the MPFV sequence at amino acid positions 54,78 , and 139 , respectively (Fig. 1a). The seven sequence motifs (i.e., I, Ia, II, III, IV, V, and VI) associated with NTP hydrolysis and nucleic acid binding ${ }^{32,33}$, were well conserved among MPFV and other flaviviruses (Fig. 1a). The alignment of NS5 sequences indicated two (1 and 2) and four (A-D) conserved motifs related to methyltransferase and RdRp activities, respectively (Fig. 1b). The two N-terminal motifs involved in RNA cap methylation at the first nucleotide of the newly synthesized positive-strand RNA ${ }^{34}$ could be mapped within the MPFV sequence, and an aspartic acid residue critical for enzymatic activity ${ }^{35}$ was present in the MPFV NS5 sequence at amino acid position 147 . Four Cterminal motifs (A-D) associated with RNA synthesis through RdRp activity were conserved among MPFV as with other flaviviruses ${ }^{36}$. Of these, the motifs $A$ and $C$ constituting the core of the catalytic site ${ }^{36}$ contained four important conserved aspartic acid residues in the MPFV NS5 sequence at the following positions: 536, 541, 665, and 666 (Fig. 1b). Additionally, the hydropathy profiles based on the NS3 and NS5 of MPFV were similar to those of tick-borne encephalitis virus (see Supplementary Fig. S1 online). These results suggest that the polyprotein processing and organization of its replication complex are functionally conserved among MPFV and other flaviviruses.

Phylogenetic analyses were conducted to investigate the evolutionary relationship among MPFV and other flaviviruses (Fig. 2). In the phylogenetic tree based on the complete consensus flavivirus polyprotein sequence (Fig. 3a), MPFV was located independently from any other known flavivirus, but shared a common origin with TBFV and TBFV-related NKV (Fig. 2). Phylogenetic analyses of E, NS3, and NS5 from MPFV and representative flaviviruses revealed that MPFV also formed a distinct branch among members of the genus Flavivirus (Figs. 3b-d). These results suggest that MPFV is the first known representation of a flavivirus that shares ancestral roots with TBFVs and TBFV-related NKVs. To clarify the phylogenetic relationship between MPFV and Ngoye virus, we constructed a 
phylogenetic tree based on 1,392 amino acid sequences from the partially identified polyprotein of Ngoye virus (Fig. 3a). MPFV clustered only with Ngoye virus, with a unique lineage being formed by MPFV and Ngoye virus (Fig. 3e). These results indicate that tick-related flaviviruses from ticks collected in Africa (i.e., Senegal and Zambia) may form a distinct flavivirus group.

\section{MPFV shares similar dinucleotide compositions with TBFVs and mimics the vertebrate genome signature}

Analysis of nucleotide and dinucleotide contents of viruses and their hosts indicates that flaviviruses have coevolved with the latter ${ }^{37,38}$. To predict the host range of MPFV based on dinucleotide usage, a linear discriminant analysis was performed based on the dinucleotide ratios in complete or nearly complete genome sequences from 128 flaviviruses with a defined host range and transmissibility, which has been previously reported to determine host specificity (Fig. 4a) ${ }^{38-41}$. MPFV was predicted as a TBFV based on five flavivirus groups via this analysis.

In addition, we compared dinucleotide ratios for 16 dinucleotides across MPFV and five flavivirus groups (Fig. 4b). As a threshold of dinucleotide patterns, Karlin and Mrazek showed that dinucleotide ratios $<0.78$ can be regarded as underrepresented dinucleotides, whereas values $>1.23$ indicate overrepresented dinucleotides ${ }^{42}$. All vertebrateinfecting flavivirus groups (i.e., NKV, MBFV, and TBFV) contain underrepresented CpG and TpA, and overrepresented $\mathrm{TpG}$ and $\mathrm{CpA}$. These characteristic dinucleotide ratios are commonly found in vertebrate-infecting viruses and mimic the vertebrate genome signature 37,38 . MPFV also exhibits these common characteristics, indicating that the virus may belong to TBFV, vertebrate-infecting flavivirus groups.

\section{MPFV has unique RNA secondary structures in its 3'-UTR}

In addition to identifying characteristic evolutionary patterns in the coding regions, we performed in silico thermodynamic modelling of the MPFV UTRs. Both UTRs are known to harbor unique RNA structural elements that are evolutionarily conserved among many flaviviruses ${ }^{30}$. The $5^{\prime}$-UTR of MPFV folds into canonical stem-loop A (SLA) and B (SLB) structures, and is immediately followed by a capsid hairpin element (cHP; Fig. 5a). The overall composition of the $5^{\prime}$-UTR resembles that of other flaviviruses ${ }^{43,44}$ with an extended SLA side-stem loop characteristic of TBFVs (unpublished data). Similarly, the MPFV 3'-UTR consists of a set of functional elements that are organized into three domains and exhibit structural homology to known elements within other flaviviruses. These include two distinct xrRNAs, a dumbbell (DB) element as well as a conserved terminal 3' stem-loop (3'SL) structure (Fig. 5b). Other stem-loops predicted in the MPFV 3'-UTR do not show homology to known functional elements. The MPFV DB element is of particular interest, given that DB elements are typically not found in the 3'-UTRs of mammalian or seabird-associated TBFVs, but are present in several TBFV-related NKVs ${ }^{30}$, with which MPFV shares ancestral roots. Although the proximal stem-loop originating from the central multi-loop of the MPFV DB element is considerably shorter than the DB elements of MBFVs or dual-host affiliated ISFVs ${ }^{30,45}$, for example, MPFV is unique among the known TBFVs to contain such an element in its 3'-UTR.

\section{MPFV xrRNA structures can block exoribonuclease in vitro}

Exoribonuclease-resistant RNAs are short RNAs, typically 60-90 nucleotides in length, with the capacity to stall exoribonucleases. They are commonly comprised of a three-way junction element, downstream hairpin-loop and transient RNA pseudoknot ${ }^{15,30}$. The xrRNAs located in flaviviral 3'-UTRs contribute to the production of sfRNAs formed by partial degradation of viral RNA via cellular 5'-3' exoribonucleases such as Xrn1. Although the RNA structures observed in the 3'-UTR of MPFV were structurally homologous to previously described xrRNAs ${ }^{23,24}$, a covariance model analysis against known xrRNAs of other known flaviviruses did not yield any plausible hits. This is 
likely due to the fact that MPFV xrRNAs are sufficiently diverged from the xrRNAs of previously described flaviviruses that have been used to construct covariance models. To confirm whether these RNA elements can stall exoribonucleases, Xrn1 degradation assays were performed in vitro. Through an RNA design approach based on RNAblueprint ${ }^{46}$, we designed two RNA constructs, consisting of potential xrRNA-forming sequences of MPFV and 31-nucleotide leader sequences that do not interact with the xrRNA three-way junction fold. The reliability of the predicted structures was assessed in terms of positional entropy, highlighting that the leader sequence is effectively unpaired (Figs. 6a and 6b). The Xrn1 degradation assays using these two RNA constructs [(+31)-xrRNA1 and (+31)xrRNA2] revealed that xrRNA1 and xrRNA2 effectively blocked the progression of Xrn1 (Fig. 6c). The precise locations at which Xrn1 is blocked by xrRNAs were mapped using a primer extension method. The Xrn1 halt site of partially degraded xrRNA1 was located in the basal stem of xrRNA1 (Figs. 6a and 6d). Conversely, xrRNA2 analysis revealed that the truncated RNA ends with an uracil, 5 nucleotides upstream of the three-way junction secondary structure (Figs. 6b and 6e). While the halt site in the xrRNA1 construct is unexpectedly located in a stem-loop structure, we attribute this phenomenon to the high conformational flexibility of the basal 3-nucleotide stem in the predicted (31)-xrRNA1 structure, as expressed by high positional entropy (Fig. 6a). On the contrary, the entire closing stem of (31)-xrRNA2 exhibits low positional entropy, indicating that this predicted structure is well defined (Fig. 6b). These results suggest that MPFV forms two unique functional xrRNA structures in the 3'-UTR, and that the sfRNA of MPFV can be produced as observed in other flaviviruses.

\section{Discussion}

In this study, we described the discovery of a novel flavivirus tentatively named MPFV, isolated from a Rhipicephalus muhsamae in Zambia and determined its whole genome sequence including the 5'-UTR, the ORF, and the 3'-UTR. MPFV was found to be closely related to the Ngoye virus previously detected in Senegal ${ }^{12}$, and these flaviviruses are phylogenetically distinct from other TBFVs. While TBFVs have been mainly detected in Europe, North America, Siberia, and Far East regions ${ }^{47}$, our results suggest that a novel TBFV group, including MPFV and Ngoye virus, may exist on the African continent. Considering the long distance (>5,000 km) between Zambia and Senegal, the discovery of MPFV and Ngoye virus suggests that TBFVs belonging to a novel TBFV group may be widely distributed in Africa. Our findings provide new information regarding the geographical distribution and genetic diversity of flaviviruses.

Unfortunately, the isolation of MPFV using cell culture and neonatal mice was unsuccessful in the present study. In a previous report, newborn mice and several cell lines were used for isolation of Ngoye virus, but viral amplification was similarly not observed as our results ${ }^{12}$. Thus, we predicted the host range of MPFV using a linear discriminant analysis based on the dinucleotide ratios. Flaviviruses mimic the dinucleotide composition of their hosts, indicating that there is a clear difference in dinucleotide composition between vertebrate-infecting and invertebrate-specific flaviviruses ${ }^{37}$. A previous study showed linear discriminant analysis for Flaviviridae with a high sensitivity rate, as $99 \%$ of vector-borne flaviviruses were accurately predicted within the current flavivirus group ${ }^{38}$. Therefore, dinucleotide compositions are thought to be a reliable tool for predicting the host range of flaviviruses. Our compositional analyses show that MPFV is predicted to be a TBFV and exhibits similar dinucleotide composition characteristics with vertebrate-infecting flaviviruses. These results suggest that MPFV may infect vertebrate hosts in the same manner as other TBFVs, which could impose a public health risk. Ngoye virus was detected in ticks captured from ovine and caprine species ${ }^{12}$, and it would be of interest to investigate the prevalence of MPFV in mammals, such as livestock, for risk estimation. Recently, novel flaviviruses were detected in marine vertebrates and invertebrates ${ }^{48-50}$, and the genome of crustacean flaviviruses-namely marine invertebrate flaviviruses-were shown 
to be related to terrestrial vector-borne flaviviruses ${ }^{48}$. Thus, improved understanding of the potential origins of invertebrate-vertebrate flaviviruses is likely necessary to explore potential vertebrate hosts of MPFV. Further epidemiological studies utilizing different approaches are required to estimate the host range and risk of novel TBFV group flaviviruses.

Both the $5^{\prime}$ - and the $3^{\prime}-$ UTRs of flaviviruses contain sequence motifs related to viral translation and replication ${ }^{16-22}$. The secondary structure and function of the 5 '-UTR and areas adjacent to the capsid coding region are well conserved among flaviviruses and contain three RNA elements with crucial functions (SLA, SLB, and cHP) ${ }^{16-21}$. The large SLA positioned in the 5 '-terminus of flaviviral genomes plays a role as a promoter element recognized by viral RdRp; binding of RdRp to SLA is necessary for viral RNA synthesis ${ }^{16,17}$. The second short SLB, often containing an initiation AUG codon, is complementary to a sequence present at the 3 '-end of the viral genome, and is also important for viral replication ${ }^{18,19}$. The $\mathrm{CHP}$ in the $\mathrm{C}$ coding region enhances recognition of the AUG codon, and is required for RNA synthesis and late translation of viral proteins ${ }^{20,21}$. Our data indicate that the MPFV genome contains a typical flavivirus 5'-UTR structure, including canonical SLA, SLB, and cHP structures. Thus, MPFV viral RNA synthesis would be expected to be conducted in the same manner as other flaviviruses. Conversely, organization of the 3'-UTR differs between flaviviruses, and sequence composition, length, and secondary structures are also considerably varied ${ }^{30}$. Nevertheless, some RNA secondary structures are shared among the flavivirus groups ${ }^{30}$. Of these, the terminal $3^{\prime} S L$ structures, which are associated with long-range RNA-RNA interactions between the $5^{\prime}$ - and the $3^{\prime}$-UTRs, are required for virus replication, and are therefore present in the 3 ' termini of all flaviviruses ${ }^{17}$. Although MPFV contained similar 3 'SL structures to other TBFVs, other secondary structures differed from those of known TBFVs. DB elements, for example, are not conserved within TBFVs, but are conserved between MBFVs and dual-host affiliated ISFV. Although the proximal stem-loop originating from the central multi-loop of the DB element in the 3'-UTR of MPFV and TBFV-related NKVs is shorter than those of MBFVs and dual-host affiliated ISFVs, the biological and functional significance of these differences remains unclear. Generally, the 3'-UTRs of most MBFVs have two DB elements, which are related to genomic cyclization and optimal translation ${ }^{22}$. Further research on genomic cyclization and translation may be required for accurate biological characterization of the shorter stemloops within DB elements in MPFV and TBFV-related NKVs. For other MPFV stem-loop structures, no evolutionary support was obtained among other flaviviruses following a bioinformatic homology search. These results indicate that the 3'-UTR of MPFV is unique and may help to the understanding of the evolutionary history of the 3'-UTR in flaviviruses.

We demonstrated that two xrRNAs derived from the 3'-UTR of MPFV achieved Xrn1 resistance through in vitro exoribonuclease assays. X-ray crystallography of xrRNA structures derived from MBFVs revealed a three-way junction and a pseudoknot interaction creating an unusual and complex fold, and forming a ring-like structure during folding ${ }^{51,52}$. The $5^{\prime}$ end of the xrRNA passes through the center of the ring-like structure, and this feature results in Xrn1 resistance to xrRNA structures. A pseudoknot interaction, which might be transient, is formed via base pairing (2-7 nucleotides) between the apical xrRNA loop and downstream sequence ${ }^{24}$. Within the xrRNA1 of MPFV, putative base pairing occurs between a 5'-UGACC-3' sequence in the apical loop and a 5'-GGUCA-3' sequence 26 nucleotides downstream; the complementary sequence of the apical loop also appears within a downstream stem-loop structure for xrRNA2 (see Supplementary Fig. S2 online). These putative pairing sequences might be related to a tertiary interaction during formation of these xrRNAs. Since these predictions are merely suggested interactions without the support of thermodynamic-based modeling, further studies of RNA structures using X-ray crystallography are required to clarify the mechanism of exoribonuclease resistance for xrRNA structures. 
In general, multiple xrRNAs are present in the 3'-UTR of flaviviruses, producing multiple sfRNAs ${ }^{25}$. Although the pattern of sfRNA production in human cells infected with West Nile virus demonstrated that the longest sfRNA was efficiently produced by the first xrRNA, the second xrRNA containing certain mutations and abrogating tertiary interactions also led to a decrease in the amount of the longest sfRNA, suggesting that xrRNA duplication in the $5^{\prime}$ end of the $3^{\prime}-U T R$ is required for efficient production of sfRNA via an unknown mechanism ${ }^{25}$. The two functional MPFV xrRNAs were located in succession near the 5' end of the 3'-UTR and might contribute to producing complete sfRNA in MPFV due to incomplete degradation of RNA by the host exoribonuclease. Duplications of xrRNA structures at the 5 ' end of the 3 '-UTR are common in vector-borne flaviviruses, such as MBFV and TBFV, and were uncommon in the $3^{\prime}$-UTRs of ISFV ${ }^{29}$. The xrRNA duplication observed in vector-borne flaviviruses is related to host adaptation without reduction of viral fitness during host switching between invertebrates and vertebrates ${ }^{25,29}$. The first xrRNA of West Nile virus, when mutated to disrupt its structure, significantly led to reduction in its pathogenicity, indicating that this xrRNA plays a role in virulence to vertebrate hosts ${ }^{28}$. Interestingly, deletion of the second xrRNA in Dengue virus led to an increase in viral replication in mosquito cells, whereas deletion of both xrRNAs reduced viral replication in mammalian cells ${ }^{29}$. In previous studies, a new model was proposed in which vector-borne flaviviruses containing xrRNA duplication maintain the potential for efficient sfRNA production by maintaining the intact first xrRNA in both vertebrates and invertebrates, but in invertebrates these viruses modulate the second xrRNA to enable adaptation to their host ${ }^{25,29}$. Therefore, it is reasonable to assume that MPFV is a vector-borne flavivirus containing duplicated xrRNA, and further exploration is needed to assess its significance.

\section{Methods}

\section{Ethical statement and Sample collection}

All animal experiments were performed with approval from the Animal Care and Use Committee of Hokkaido University following the Fundamental Guidelines for Proper Conduct of Animal Experiment and Related Activities in Academic Research Institutions under the jurisdiction of the Ministry of Education, Culture, Sports, Science and Technology in Japan (permit number 19-0019). Adult host-questing ticks were captured using the flagging method, and blood-sucking ticks were collected from domestic animals (e.g., cattle, goat, sheep, and dogs) in Isoka (10.15 ${ }^{\circ} \mathrm{S}$, $\left.32.63^{\circ} \mathrm{E}\right)$, Mpulungu $\left(8.76^{\circ} \mathrm{S}, 31.11^{\circ} \mathrm{E}\right)$, and Samfya $\left(11.36^{\circ} \mathrm{S}, 29.55^{\circ} \mathrm{E}\right)$ from November 2017 to January 2018 in Zambia. Collected ticks ( $n=573$ ) were morphologically identified under a stereomicroscope (see Supplementary Table S1 online). Each tick was washed in $70 \%$ ethanol containing $1 \%$ iodine and then submerged in distilled water. The samples were then homogenized with $200 \mu$ of Dulbecco's modified Eagle's medium using a homogenizer (Tomy Seiko, Tokyo, Japan) at 3,000 rpm. Total RNAs were extracted from $100 \mu \mathrm{l}$ of the homogenates using TRIzolLS (Invitrogen, Waltham, MA) according to the manufacturer's protocol, and remaining lysate samples were stored at $-80^{\circ} \mathrm{C}$ until use for virus isolation.

\section{Detection of flavivirus}

Tick RNA samples were examined to detect flavivirus via RT-PCR using a One Step RT-PCR Kit v2 (Takara, Shiga, Japan) with pan-flavivirus primer set (see Supplementary Table S2 online) based on the conserved sequence within the flavivirus NS5 protein as previously described ${ }^{53}$. The RT-PCR conditions were as follows: initial reverse transcription step at $50^{\circ} \mathrm{C}$ for $30 \mathrm{~min}$; PCR activation step at $94^{\circ} \mathrm{C}$ for $2 \mathrm{~min} ; 43$ cycles of $94^{\circ} \mathrm{C}$ for $30 \mathrm{~s}, 53^{\circ} \mathrm{C}$ for $30 \mathrm{~s}$, and $72^{\circ} \mathrm{C}$ for $30 \mathrm{~s}$; and a final extension at $72^{\circ} \mathrm{C}$ for $5 \mathrm{~min}$. PCR products were subjected to direct sequencing using the Big Dye Terminator v3.1 Cycle Sequencing Kit (Applied Biosystems, Foster City, CA). 


\section{Virus isolation}

Tick homogenates positive for flavivirus were cultured in Vero E6 (kindly provided by Dr. Heinz Feldmann, National Institutes of Health, Bethesda, MD), BHK-21 (gift from Dr. Akira Oya, the National Institutes of Health, now National Institute of Infectious Diseases, Tokyo, Japan), ISE6 (kindly provided by Dr. Ulrike Munderloh, University of Minnesota, Saint Paul, MN) or C6/36 cells (purchased from the American Type Culture Collection, Manassas, VA). Tick lysates were inoculated into these cell lines, and supernatants and cell lysates were examined at each passage for detection of flavivirus genome by RT-PCR of NS5. Lysates were also inoculated into neonatal mice brain via intracerebral injections, and brain-derived RNA was subsequently subjected to RT-PCR.

\section{Library preparation and whole genome sequencing}

Total RNAs extracted from tick homogenates positive for flavivirus were used for whole genome sequencing. Ribosomal RNA depletion from total RNA was performed using RiboMinus Eukaryote Kit for RNA-Seq (Invitrogen), and cDNA was synthesized using a PrimeScript Double Strand cDNA Synthesis Kit (Takara) according to the manufacturers' instructions. The cDNA libraries were prepared using a Nextera XT DNA Library Preparation Kit (Illumina, San Diego, CA) according to the manufacturer's instructions, and were then subjected to whole-genome sequencing on a MiSeq using a MiSeq Reagent Kit v3 (600 cycles) (Illumina). Sequencing data was analyzed using the CLC Genomics Workbench software (CLC bio, Hilden, Germany). Flavivirus genome contigs were obtained by de novo assembly and the overlapped contig sequences were confirmed by PCR amplification with specific primers and Sanger sequencing. The $5^{\prime}$ and $3^{\prime}$ termini of the flavivirus genome were amplified using RACE with specific primers and a SMARTer RACE cDNA Amplification Kit (Takara) according to the manufacturer's protocol (see Supplementary Table S2 online). Amplified products were directly sequenced using a BigDye Terminator v3.1 Cycle Sequencing Kit.

\section{Genetic comparison and phylogenetic analyses of flaviviruses}

Polyprotein ORF positions were predicted using GENETYX version 12 (GENETYX Corporation, Tokyo, Japan). The putative cleavage sites of the detected flavivirus were determined by comparison with known cleavage sites of previously characterized flaviviruses as well as cleavage patterns of a host signal peptidase, furin and viral serine protease as previously described ${ }^{54}$. Bioinformatic analyses were performed using flavivirus sequences deposited in the DDBJ/EMBL-Bank/GenBank databases. Identity comparison analyses were conducted among flaviviruses using GENETYX version 12. Conserved enzymatic motifs of NS3 and NS5 were identified by sequence alignments of detected and previously characterized flaviviruses. The hydropathy profiles of viral proteins were obtained using the web-based tool ProtScale (https://web.expasy.org/protscale/) from the ExPASy Bioinformatics Resource Portal with the Kyte and Doolittle scale option ${ }^{55}$. Phylogenetic analyses based on the amino acid sequence of flavivirus polyprotein and each viral protein were performed using MEGA7 ${ }^{56}$. The MUSCLE protocol was used to align the sequences, and phylogenetic trees were constructed using the maximum-likelihood method based on the TamuraNei model with 1,000 bootstrap replicates.

\section{Compositional analysis}

Complete or nearly complete genome sequences from 128 flaviviruses with defined host range and transmissibility were used as a dataset, which were classified as TBFV, MBFV, NKV, ISFV, and flaviviruses derived from marine organisms (i.e., Marine), and used for analyses (see Supplementary Table S4 online). The dinucleotide ratios (observed/expected values) were calculated using the formula $P_{X Y}=f_{X Y} / f_{X} f_{Y}$, in which $f_{X}$ and $f_{Y}$ denote the 
frequencies of the mononucleotides $X$ and $Y$, respectively, and $f_{X Y}$ denotes the frequency of dinucleotide $X Y^{42}$. Linear discriminant analysis was performed using the R package (The R Project for Statistical Computing, 2018).

\section{Prediction of RNA secondary structure}

RNA secondary structure predictions in both MPFV UTRs were computed using RNAfold of the Vienna RNA Package ${ }^{57}$, explicitly disallowing isolated base pairs (--noLP option). Structural homology of the predicted 5'-UTR stem-loop structures SLA and SLB to these elements in other flaviviruses was determined by Infernal covariance models $(\mathrm{CMs})^{58}$. Likewise, TBFV-specific CMs from a recent study ${ }^{30}$ were used to confirm the predicted locus of the MPLV 3 'SL element, and the Rfam ${ }^{59}$ CM RF00525 (Flavivirus_DB) was used to annotate the single DB element in the 3'-UTR. All secondary structure plots were produced with the RNAplot utility ${ }^{57}$.

\section{Design of leader sequences for the Xrn 1 degradation assay}

To test the capacity of the predicted 3'-UTR structures xrRNA1 and xrRNA2 to inhibit nuclease digestion, we performed an Xrn1 degradation assay according to the methods of Chapman et al. ${ }^{23}$. To this end, we extracted the nucleotide sequences folding into three-way junction structures together with their downstream hairpins. The degradation assay requires a leader sequence upstream of the xrRNA to load Xrn1. To exclude the possibility that the leader sequence interacts with the sequence forming the three-way junction, we designed custom, artificial $31 \mathrm{nt}$ leader sequences that do not form any significant secondary structures nor distort the canonical xrRNA fold. We sampled sequences fulfilling this requirement using RNAblueprint ${ }^{46}$. We employed partition function folding with default parameters implemented in the ViennaRNA package to obtain ensemble free energies $\Delta G$, optimizing for a maximal $\left(Z^{F} / Z\right)$ ratio as design goal, where $Z^{F}$ is the partition function under the constraint that the three-way junction and downstream hairpin are formed in the presence of the designed $31 \mathrm{nt}$ leader sequence, and $Z$ is the unconstrained partition function. $Z$ is related to the ensemble free energy via $\Delta G=-R T I n Z$, where $R$ is the universal gas constant and $\mathrm{T}$ is the thermodynamic temperature. Reliability of the predicted structures, including the designed leader sequences was, visualized in terms of positional entropy (Figs. 6a and 6b).

\section{Xrn1 degradation assay}

Two 3'-UTR RNAs [(+31)-xrRNA1 and (+31)-xrRNA2] were chemically synthesized and purified by the Agilent 1290 Infinity II chromatography system (Agilent Technologies, Santa Clara, CA; see Supplementary Table S5 online). The synthesized RNAs were phosphorylated using T4 Polynucleotide Kinase (Takara) and purified by the RNeasy MinElute Cleanup Kit (Qiagen, Hilden, Germany) according to the manufacturer's instructions. The modified RNAs were incubated at $90^{\circ} \mathrm{C}$ for $2 \mathrm{~min}$, followed by $20^{\circ} \mathrm{C}$ for $5 \mathrm{~min}$, then held at $4^{\circ} \mathrm{C}$ for RNA folding. Xrn 1 digestion reactions were conducted with 3-4 $\mu \mathrm{g}$ of the 3'-UTR RNA ( $100 \mathrm{pmol})$ and $2 \mu \mathrm{g}$ of 5 ' monophosphorylated 31-mer control RNA ( 200 pmol) in NEB3 buffer [100 mM NaCl, $50 \mathrm{mM}$ Tris- $\mathrm{HCl}(\mathrm{pH} 7.9), 10 \mathrm{mM} \mathrm{MgCl}$, and $1 \mathrm{mM} \mathrm{DTT]}$ (New England Biolabs, Ipswich, MA). The RNA mixture was split between two tubes, and two units of Xrn1 (New England Biolabs) were added to one aliquot while the other served as an Xrn1-negative control. Both mixtures were incubated at $37^{\circ} \mathrm{C}$ for $2 \mathrm{~h}$, followed by quenching via addition of an equal volume of Novex TBE-Urea Sample Buffer (Invitrogen). The RNA products were analyzed on 15\% denaturing PAGE gel and visualized by ethidium bromide staining.

\section{Mapping Xrn1 halt sites of 3'-UTR RNAs}


Xrn1 halt sites were mapped by RNA sequencing utilizing the primer extension method ${ }^{60}$. RNA products remaining after the Xrn1 degradation assay were recovered using the ZR small-RNA PAGE Recovery Kit (ZYMO research, Irvine, $\mathrm{CA}$ ) according to the manufacturer's instruction. Reverse transcription was carried out using SuperScript IV Reverse Transcriptase (Invitrogen) according to the manufacturer's instruction. Briefly, approximately 2 pmol of recovered RNA products were annealed to the $5^{\prime}$ end-labeled primer with deoxyribonucleoside triphosphates and dideoxynucleoside triphosphates (see Supplementary Table S2 online). Reverse transcriptase and buffer components were added to the primer-RNA hybrids to catalyze elongation of the primer to 5 ' end of the RNA. Following the elongation reaction, equal volumes of loading buffer ( $95 \%$ formamide and $10 \mathrm{mM}$ EDTA) were added to the reactions, and the mixtures were then incubated at $80^{\circ} \mathrm{C}$ for $5 \mathrm{~min}$. The resulting fluorescein-labeled cDNA products were analyzed on a 15\% denaturing PAGE gel and visualized by the ChemiDoc Touch Imaging System (BioRad Laboratories, Inc., Hercules, CA).

\section{Data Availability Statement}

The sequence of Mpulungu flavivirus was deposited into the GenBank/EMBL/DDBJ database (Accession No. LC582740). All data generated or analyzed within this study are included in this published manuscript and its supplementary information files.

\section{Declarations}

\section{Acknowledgements}

We would like to thank Mr. Kenji Yokoi for coordinating the sampling activity and Ms. Aiko Ohnuma and Ms. Chiaki Funaki for the technical supports of analyses. We also thank Mr. Evans Mulenga, Mr. Kapila Penjaninge, Mr. John Chilundu and the veterinary staffs at Ministry of Fisheries and Livestock for collecting samples. This work was supported by grants for Scientific Research (B) from the Ministry of Education, Culture, Sports, Science and Technology, Japan (MEXT) / Japan Society for the Promotion of Science (JSPS) KAKENHI (JP16H05805); and grants for Scientific Research on Innovative Areas and International Group from the MEXT/JSPS KAKENHI (JP16H06431, JP16H06429, JP16K21723); and the Japan Initiative for Global Research Network of Infectious Diseases (JP15fm0108008) and the Japan Program for Infectious Diseases Research and Infrastructure (JP20wm0125008) from Japan Agency for Medical Research and Development (AMED); and grants for the AMED and Japan International Cooperation Agency (JICA) within the framework of the Science and Technology Research Partnership for Sustainable Development (SATREPS) (JP19jm0110019).

\section{Author contributions statement}

H.H. conceived and designed the experiments; H.H., Y.O., S.T., Y.Q., M.K., Yo.E., B.M.H., Yu.E., Ma.Si., and H.S. collected samples; H.H., Y.O., and S.T. performed the experiments; H.H., N.M., T.A., W.W.H. and M.W. analyzed the data; K.U., Mi.Sa., K.Y., R.N., and K.M. advised for the experiments; H.H. wrote the original draft of manuscript. T.A. and M.W. rewrote parts of the manuscript. All authors edited and approved the manuscript.

\section{Competing interests}

The authors declare no competing interests.

\section{References}


1. Pierson, T. C. \& Diamond, M. S. Flaviviruses in Fields Virology vol 2, 6th Edn. (eds. Knipe, D.M., Howley, P.M., Cohen, J.I., Griffın, D.E., Lamb, R.A., Martin, M.A., Racaniello, V.R., Roizman, B.) 747-794 (Lippincott Williams \& Wilkins, 2013).

2. Pierson, T. C. \& Diamond, M. S. The continued threat of emerging flaviviruses. Nat Microbio/5, 796-812, doi:10.1038/s41564-020-0714-0 (2020).

3. Simmonds, P. et al. ICTV Virus Taxonomy Profile: Flaviviridae. J Gen Viro/98, 2-3, doi:10.1099/jgv.0.000672 (2017).

4. L'vov, D. K. et al. [Kama, a new virus (Flaviviridae, Flavivirus, Tiulenii antigenic group), isolated from Ixodes lividus ticks]. Vopr Viruso/43, 71-74 (1998).

5. Lvov, D. K. et al. "Karshi" virus, a new flavivirus (Togaviridae) isolated from Ornithodoros papillipes (Birula, 1895) ticks in Uzbek S.S.R. Arch Viro/50, 29-36, doi:10.1007/BF01317998 (1976).

6. Zaki, A. M. Isolation of a flavivirus related to the tick-borne encephalitis complex from human cases in Saudi Arabia. Trans R Soc Trop Med Hyg91, 179-181, doi:10.1016/s0035-9203(97)90215-7 (1997).

7. Carletti, F. et al. Alkhurma hemorrhagic fever in travelers returning from Egypt, 2010. Emerg Infect Dis16, 19791982, doi:10.3201/eid1612.101092 (2010).

8. Musso, M., Galati, V., Stella, M. C. \& Capone, A. A case of Alkhumra virus infection. J Clin Viro/66, 12-14, doi:10.1016/j.jcv.2015.02.019 (2015).

9. Horton, K. C. et al. Crimean Congo Hemorrhagic Fever Virus and Alkhurma (Alkhumra) Virus in Ticks in Djibouti. Vector Borne Zoonotic Dis16, 680-682, doi:10.1089/vbz.2016.1951 (2016).

10. Davies, F. G. Nairobi sheep disease in Kenya. The isolation of virus from sheep and goats, ticks and possible maintenance hosts. J Hyg (Lond)81, 259-265, doi:10.1017/s0022172400025092 (1978).

11. Mugo, W. N. \& Shope, R. E. Kadam virus: neutralization studies and laboratory transmission by Dermacentor variabilis. Trans R Soc Trop Med Hyg66, 300-304, doi:10.1016/0035-9203(72)90162-9 (1972).

12. Grard, G. et al. Ngoye virus: a novel evolutionary lineage within the genus Flavivirus. J Gen Viro/87, 3273-3277, doi:10.1099/vir.0.82071-0 (2006).

13. Chambers, T. J., McCourt, D. W. \& Rice, C. M. Production of yellow fever virus proteins in infected cells: identification of discrete polyprotein species and analysis of cleavage kinetics using region-specific polyclonal antisera. Virology177, 159-174, doi:10.1016/0042-6822(90)90470-c (1990).

14. Rice, C. M. et al. Nucleotide sequence of yellow fever virus: implications for flavivirus gene expression and evolution. Science229, 726-733, doi:10.1126/science.4023707 (1985).

15. Ng, W. C., Soto-Acosta, R., Bradrick, S. S., Garcia-Blanco, M. A. \& Ooi, E. E. The 5' and 3' Untranslated Regions of the Flaviviral Genome. Viruses9, doi:10.3390/v9060137 (2017).

16. Yu, L., Nomaguchi, M., Padmanabhan, R. \& Markoff, L. Specific requirements for elements of the $5^{\prime}$ and $3^{\prime}$ terminal regions in flavivirus RNA synthesis and viral replication. Virology374, 170-185, doi:10.1016/j.virol.2007.12.035 (2008).

17. Filomatori, C. V. et al. A 5 ' RNA element promotes dengue virus RNA synthesis on a circular genome. Genes Dev20, 2238-2249, doi:10.1101/gad.1444206 (2006).

18. Alvarez, D. E., Filomatori, C. V. \& Gamarnik, A. V. Functional analysis of dengue virus cyclization sequences located at the 5' and 3'UTRs. Virology375, 223-235, doi:10.1016/j.virol.2008.01.014 (2008).

19. Alvarez, D. E., Lodeiro, M. F., Ludueña, S. J., Pietrasanta, L. I. \& Gamarnik, A. V. Long-range RNA-RNA interactions circularize the dengue virus genome. J Viro/79, 6631-6643, doi:10.1128/JVI.79.11.6631-6643.2005 (2005).

Page 13/24 
20. Clyde, K. \& Harris, E. RNA secondary structure in the coding region of dengue virus type 2 directs translation start codon selection and is required for viral replication. J Viro/80, 2170-2182, doi:10.1128/JVI.80.5.21702182.2006 (2006).

21. Clyde, K., Barrera, J. \& Harris, E. The capsid-coding region hairpin element (cHP) is a critical determinant of dengue virus and West Nile virus RNA synthesis. Virology379, 314-323, doi:10.1016/j.virol.2008.06.034 (2008).

22. Manzano, M. et al. Identification of cis-acting elements in the 3 '-untranslated region of the dengue virus type 2 RNA that modulate translation and replication. J Biol Chem286, 22521-22534, doi:10.1074/jbc.M111.234302 (2011).

23. Chapman, E. G., Moon, S. L., Wilusz, J. \& Kieft, J. S. RNA structures that resist degradation by Xrn1 produce a pathogenic Dengue virus RNA. Elife3, e01892, doi:10.7554/eLife.01892 (2014).

24. MacFadden, A. et al. Mechanism and structural diversity of exoribonuclease-resistant RNA structures in flaviviral RNAs. Nat Commun9, 119, doi:10.1038/s41467-017-02604-y (2018).

25. Kieft, J. S., Rabe, J. L. \& Chapman, E. G. New hypotheses derived from the structure of a flaviviral Xrn1-resistant RNA: Conservation, folding, and host adaptation. RNA Bio/12, 1169-1177, doi:10.1080/15476286.2015.1094599 (2015).

26. Sakai, M., Muto, M., Hirano, M., Kariwa, H. \& Yoshii, K. Virulence of tick-borne encephalitis virus is associated with intact conformational viral RNA structures in the variable region of the 3'-UTR. Virus Res203, 36-40, doi:10.1016/j.virusres.2015.03.006 (2015).

27. Roby, J. A., Pijlman, G. P., Wilusz, J. \& Khromykh, A. A. Noncoding subgenomic flavivirus RNA: multiple functions in West Nile virus pathogenesis and modulation of host responses. Viruses6, 404-427, doi:10.3390/v6020404 (2014).

28. Zhang, Q. Y. et al. Short Direct Repeats in the 3' Untranslated Region Are Involved in Subgenomic Flaviviral RNA Production. J Viro/94, doi:10.1128/JVI.01175-19 (2020).

29. Villordo, S. M., Filomatori, C. V., Sánchez-Vargas, I., Blair, C. D. \& Gamarnik, A. V. Dengue virus RNA structure specialization facilitates host adaptation. PLoS Pathog11, e1004604, doi:10.1371/journal.ppat.1004604 (2015).

30. Ochsenreiter, R., Hofacker, I. L. \& Wolfinger, M. T. Functional RNA Structures in the 3'UTR of Tick-Borne, InsectSpecific and No-Known-Vector Flaviviruses. Viruses11, doi:10.3390/v11030298 (2019).

31. Bazan, J. F. \& Fletterick, R. J. Detection of a trypsin-like serine protease domain in flaviviruses and pestiviruses. Virology171, 637-639, doi:10.1016/0042-6822(89)90639-9 (1989).

32. Wu, J., Bera, A. K., Kuhn, R. J. \& Smith, J. L. Structure of the Flavivirus helicase: implications for catalytic activity, protein interactions, and proteolytic processing. J Viro/79, 10268-10277, doi:10.1128/JVI.79.16.1026810277.2005 (2005).

33. Gorbalenya, A. E. \& Koonin, E. V. Helicases: amino acid sequence comparisons and structure-function relationships. Current opinion in structural biology3, 419-429 (1993).

34. Dong, H. et al. Flavivirus RNA methylation. J Gen Viro/95, 763-778, doi:10.1099/vir.0.062208-0 (2014).

35. Zhou, Y. et al. Structure and function of flavivirus NS5 methyltransferase. J Viro/81, 3891-3903, doi:10.1128/JVI.02704-06 (2007).

36. Selisko, B., Papageorgiou, N., Ferron, F. \& Canard, B. Structural and Functional Basis of the Fidelity of Nucleotide Selection by Flavivirus RNA-Dependent RNA Polymerases. Viruses10, doi:10.3390/v10020059 (2018). 
37. Lobo, F. P. et al. Virus-host coevolution: common patterns of nucleotide motif usage in Flaviviridae and their hosts. PLoS One4, e6282, doi:10.1371/journal.pone.0006282 (2009).

38. Di Giallonardo, F., Schlub, T. E., Shi, M. \& Holmes, E. C. Dinucleotide Composition in Animal RNA Viruses Is Shaped More by Virus Family than by Host Species. J Viro/91, doi:10.1128/JVI.02381-16 (2017).

39. Colmant, A. M. G. et al. A New Clade of Insect-Specific Flaviviruses from Australian. mSphere2, doi:10.1128/mSphere.00262-17 (2017).

40. Simón, D., Fajardo, A., Sóñora, M., Delfraro, A. \& Musto, H. Host influence in the genomic composition of flaviviruses: A multivariate approach. Biochem Biophys Res Commun492, 572-578, doi:10.1016/j.bbrc.2017.06.088 (2017).

41. Torii, S. et al. Discovery of Mwinilunga alphavirus: A novel alphavirus in Culex mosquitoes in Zambia. Virus Res250, 31-36, doi:10.1016/j.virusres.2018.04.005 (2018).

42. Karlin, S. \& Mrázek, J. Compositional differences within and between eukaryotic genomes. Proc Natl Acad Sci U $S$ A94, 10227-10232, doi:10.1073/pnas.94.19.10227 (1997).

43. Kofler, R. M., Hoenninger, V. M., Thurner, C. \& Mandl, C. W. Functional analysis of the tick-borne encephalitis virus cyclization elements indicates major differences between mosquito-borne and tick-borne flaviviruses. J Viro/80, 4099-4113, doi:10.1128/JVI.80.8.4099-4113.2006 (2006).

44. Mrozowich, T., Henrickson, A., Demeler, B. \& Patel, T. R. Nanoscale Structure Determination of Murray Valley Encephalitis and Powassan Virus Non-Coding RNAs. Viruses12, doi:10.3390/v12020190 (2020).

45. Wastika, C. E. et al. Discoveries of Exoribonuclease-Resistant Structures of Insect-Specific Flaviviruses Isolated in Zambia. Viruses12, doi:10.3390/v12091017 (2020).

46. Hammer, S., Tschiatschek, B., Flamm, C., Hofacker, I. L. \& Findeiß, S. RNAblueprint: flexible multiple target nucleic acid sequence design. Bioinformatics33, 2850-2858, doi:10.1093/bioinformatics/btx263 (2017).

47. Lindqvist, R., Upadhyay, A. \& Överby, A. K. Tick-Borne Flaviviruses and the Type I Interferon Response. Viruses10, doi:10.3390/v10070340 (2018).

48. Parry, R. \& Asgari, S. Discovery of Novel Crustacean and Cephalopod Flaviviruses: Insights into the Evolution and Circulation of Flaviviruses between Marine Invertebrate and Vertebrate Hosts. J Viro/93, doi:10.1128/JVI.00432-19 (2019).

49. Skoge, R. H., Brattespe, J., Økland, A. L., Plarre, H. \& Nylund, A. New virus of the family Flaviviridae detected in lumpfish (Cyclopterus lumpus). Arch Viro/163, 679-685, doi:10.1007/s00705-017-3643-3 (2018).

50. Shi, M. et al. The evolutionary history of vertebrate RNA viruses. Nature556, 197-202, doi:10.1038/s41586-0180012-7 (2018).

51. Akiyama, B. M. et al. Zika virus produces noncoding RNAs using a multi-pseudoknot structure that confounds a cellular exonuclease. Science354, 1148-1152, doi:10.1126/science.aah3963 (2016).

52. Chapman, E. G. et al. The structural basis of pathogenic subgenomic flavivirus RNA (sfRNA) production. Science344, 307-310, doi:10.1126/science.1250897 (2014).

53. Patel, P. et al. Development of one-step quantitative reverse transcription PCR for the rapid detection of flaviviruses. Virol J10, 58, doi:10.1186/1743-422X-10-58 (2013).

54. Blitvich, B. J. \& Firth, A. E. A Review of Flaviviruses that Have No Known Arthropod Vector. Viruses9, doi:10.3390/v9060154 (2017).

55. Gasteiger, E. et al. Protein Identification and Analysis Tools on the ExPASy Server in The proteomics protocols handbook (ed. Walker, J.M.) 571-607 (Humana Press, 2005). 
56. Kumar, S., Stecher, G. \& Tamura, K. MEGA7: Molecular Evolutionary Genetics Analysis Version 7.0 for Bigger Datasets. Mol Biol Evo/33, 1870-1874, doi:10.1093/molbev/msw054 (2016).

57. Lorenz, R. et al. ViennaRNA Package 2.0. Algorithms Mol Bio/6, 26, doi:10.1186/1748-7188-6-26 (2011).

58. Nawrocki, E. P. \& Eddy, S. R. Infernal 1.1: 100-fold faster RNA homology searches. Bioinformatics29, 2933-2935, doi:10.1093/bioinformatics/btt509 (2013).

59. Kalvari, I. et al. Rfam 13.0: shifting to a genome-centric resource for non-coding RNA families. Nucleic Acids Res46, D335-D342, doi:10.1093/nar/gkx1038 (2018).

60. Boorstein, W. R. \& Craig, E. A. Primer extension analysis of RNA. Methods Enzymo/180, 347-369, doi:10.1016/0076-6879(89)80111-9 (1989).

\section{Tables}

Table 1 Genome organization of Mpulungu flavivirus

\begin{tabular}{|lllll|}
\hline Gene & Protein & Genome position & Length (nucleotide) & Length (amino acid) \\
\hline 5'-UTR & - & $1-136$ & 136 & - \\
\hline ORF & Polyprotein & $137-10,342$ & 10,206 & 3,401 \\
\hline & C & $137-385$ & 249 & 83 \\
\hline CTHD & $386-439$ & 54 & 18 \\
\hline PrM & $440-931$ & 492 & 164 \\
\hline M & $707-931$ & 225 & 75 \\
\hline E & $932-2,419$ & 1,488 & 496 \\
\hline NS1 & $2,420-3,463$ & 1,044 & 348 \\
\hline NS2A & $3,464-4,162$ & 699 & 233 \\
\hline NS2B & $4,163-4,567$ & 405 & 135 \\
\hline NS3 & $4,568-6,421$ & 1,854 & 618 \\
\hline NS4A & $6,422-6,799$ & 378 & 126 \\
\hline 2K & $6,800-6,868$ & 69 & 23 \\
\hline NS4B & $6,869-7,630$ & 762 & - \\
\hline NS5 & $7,631-10,339$ & 2,709 & 254 \\
\hline & $10,343-10,868$ & 526 & 23 \\
\hline
\end{tabular}

The immature $\mathrm{C}$ protein consists of mature virion $\mathrm{C}$ and a $\mathrm{C}$-terminal hydrophobic domain. The precursor membrane protein undergoes cleavage by protease, resulting in a mature virion.

Abbreviations: UTR, untranslated region; ORF, open reading frame; C, mature virion C; CTHD, C-terminal hydrophobic domain; prM, precursor of membrane protein; M, membrane protein; E, envelope protein; NS, non-structural protein. 


\begin{tabular}{|c|c|c|c|c|c|c|c|c|c|c|c|}
\hline \multirow[t]{2}{*}{ Virus } & \multicolumn{11}{|c|}{ Amino acid sequence identity to Mpulungu flavivirus (\%) } \\
\hline & $\mathrm{C}$ & PrM & $\mathrm{E}$ & NS1 & NS2A & NS2B & NS3 & NS4A & NS4B & NS5 & Polyprotein \\
\hline $\begin{array}{l}\text { Louping ill } \\
\text { virus }\end{array}$ & 23.4 & 37.8 & 46.6 & 53.3 & 28.7 & 16.7 & 44.5 & 39.0 & 29.2 & 54.8 & 44.2 \\
\hline $\begin{array}{l}\text { Tick borne } \\
\text { encephalitis } \\
\text { virus }\end{array}$ & 20.9 & 37.4 & 46.2 & 52.1 & 30.5 & 19.8 & 44.7 & 37.3 & 29.6 & 56.2 & 44.5 \\
\hline $\begin{array}{l}\text { Omsk } \\
\text { hemorrhagic } \\
\text { fever virus }\end{array}$ & 18.0 & 37.1 & 46.6 & 51.5 & 27.8 & 19.0 & 44.2 & 37.0 & 29.6 & 56.6 & 44.2 \\
\hline Langat virus & 22.2 & 40.4 & 44.7 & 50.4 & 23.4 & 17.5 & 43.4 & 37.0 & 30.7 & 55.5 & 43.4 \\
\hline $\begin{array}{l}\text { Alkhurma } \\
\text { virus }\end{array}$ & 30.1 & 38.0 & 32.0 & 51.0 & 26.9 & 23.8 & 43.9 & 41.4 & 31.6 & 56.6 & 44.9 \\
\hline $\begin{array}{l}\text { Kyasanur } \\
\text { forest } \\
\text { disease } \\
\text { virus }\end{array}$ & 30.1 & 38.0 & 31.6 & 50.7 & 26.1 & 20.6 & 44.0 & 39.8 & 31.2 & 56.5 & 44.6 \\
\hline $\begin{array}{l}\text { Powassan } \\
\text { virus }\end{array}$ & 29.2 & 32.9 & 45.0 & 50.7 & 20.7 & 21.5 & 43.9 & 42.7 & 28.3 & 56.0 & 43.4 \\
\hline $\begin{array}{l}\text { Deer tick } \\
\text { virus }\end{array}$ & 28.0 & 31.7 & 45.9 & 50.7 & 20.2 & 21.5 & 44.0 & 40.3 & 29.8 & 55.7 & 43.3 \\
\hline Kadam virus & 21.9 & 35.5 & 46.7 & 47.8 & 24.6 & 27.4 & 41.2 & 34.1 & 27.7 & 55.2 & 42.8 \\
\hline $\begin{array}{l}\text { Meaban } \\
\text { virus }\end{array}$ & 28.3 & 33.1 & 44.6 & 51.2 & 26.2 & 19.6 & 42.2 & 34.4 & 29.8 & 57.4 & 43.6 \\
\hline $\begin{array}{l}\text { Saumarez } \\
\text { Reef virus }\end{array}$ & 18.0 & 36.8 & 46.3 & 51.5 & 18.9 & 17.4 & 41.4 & 35.4 & 28.2 & 56.4 & 43.0 \\
\hline Apoi virus & 29.1 & 30.0 & 34.7 & 36.4 & 18.0 & 16.9 & 40.9 & 20.1 & 21.6 & 53.5 & 28.4 \\
\hline $\begin{array}{l}\text { Rio Bravo } \\
\text { virus }\end{array}$ & 15.8 & 29.6 & 37.8 & 38.9 & 17.4 & 16.1 & 39.0 & 25.0 & 24.5 & 54.1 & 38.1 \\
\hline Zika virus & 15.6 & 32.5 & 40.2 & 44.2 & 17.4 & 19.0 & 41.0 & 28.5 & 22.3 & 58.4 & 40.7 \\
\hline $\begin{array}{l}\text { West Nile } \\
\text { virus }\end{array}$ & $<10$ & 30.0 & 38.2 & 41.6 & 19.7 & 23.6 & 40.6 & 28.0 & 24.7 & 59.2 & 40.4 \\
\hline $\begin{array}{l}\text { Yellow fever } \\
\text { virus }\end{array}$ & 25.6 & 30.6 & 42.0 & 43.9 & 27.4 & 23.2 & 42.0 & 31.7 & 29.4 & 56.3 & 42.2 \\
\hline Yokose virus & 21.9 & 35.5 & 39.1 & 42.1 & 20.9 & 24.4 & 38.3 & 28.0 & 26.6 & 54.6 & 39.9 \\
\hline $\begin{array}{l}\text { Cell fusing } \\
\text { agent virus }\end{array}$ & 16.8 & $<10$ & $<10$ & 29.3 & NA & NA & 34.6 & 12.5 & $<10$ & 45.8 & 22.2 \\
\hline $\begin{array}{l}\text { Kamiti River } \\
\text { virus }\end{array}$ & 16.6 & 11.1 & 12.9 & 24.5 & NA & NA & 31.9 & $<10$ & $<10$ & 46.9 & 25.9 \\
\hline $\begin{array}{l}\text { Tamana bat } \\
\text { virus }\end{array}$ & 14.4 & $<10$ & 12.7 & 12.8 & NA & NA & 21.8 & 21.4 & $<10$ & 23.1 & 14.0 \\
\hline Ngoye virus & NA & NA & NA & NA & NA & NA & $94.4^{*}$ & 96.8 & 93.3 & $95.8 *$ & $95.0 *$ \\
\hline
\end{tabular}


CTHD and $2 \mathrm{~K}$ protein sequences were not analyzed. ${ }^{*}$ Partial amino acid sequences were used for analyses. NA: Not analyzed

\section{Figures}

(a)

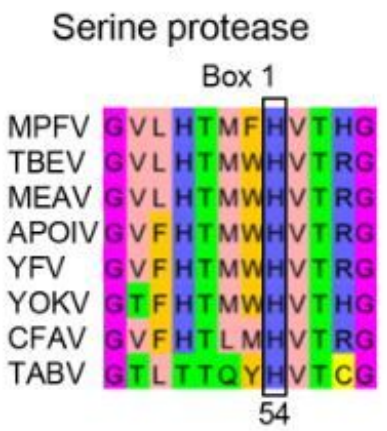

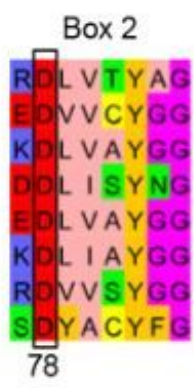

(b)

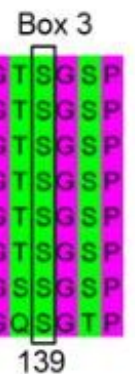

139

Helicase/NTPase

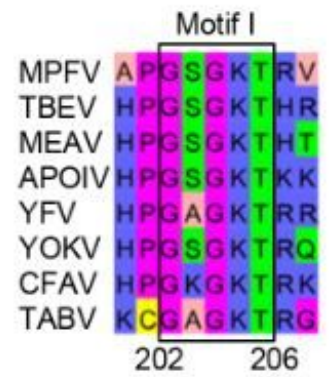

Motif II

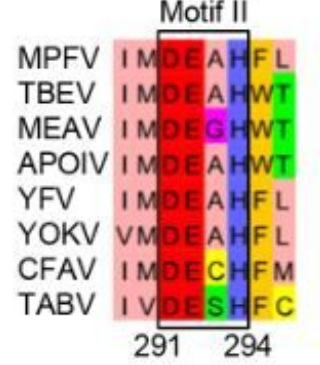

Motif V

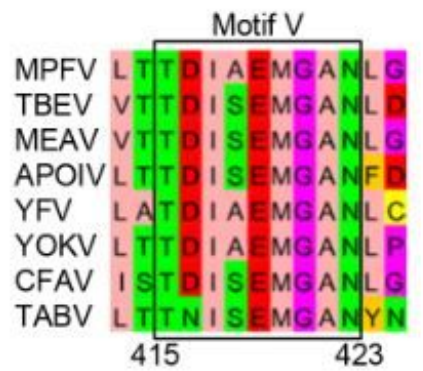

Motif la

LVLAPTRVVL DEMC
LVLAPTRVVLKEM
LVLAPTRVVLREM
LVLAPTRVVVKEM
LVLAPTRVVLSEMK
LVLAPTKVVLSEMH
V I LTPTRVVMAEVV
LVLVPTRVVANEA
229

Motif III
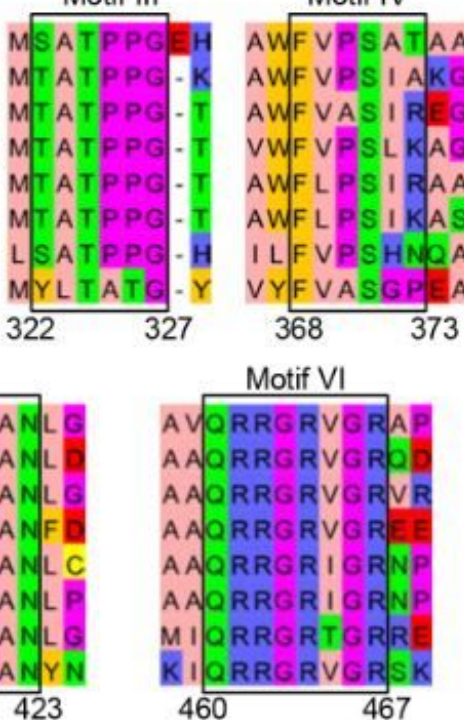

\section{Methyltransferase}
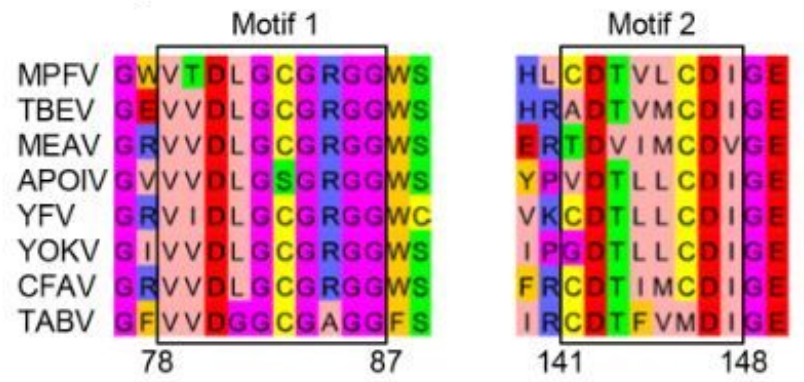

RNA-dependent RNA polymerase

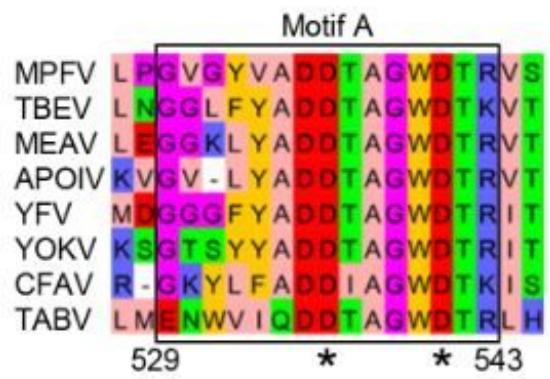

Motif B

MPFV TDVISRRDQRGSGQVVTYALNTWTN I KVQLL TBEV MDV ITRRDORGSGQVVTYALNTL TN I KVOL I MEAV M I I SRRDQRGSGQVVTYALNT I TN I KVQL I APOIVMDVISRKDQRGSGQVVTYALNTLTN I KVQLV YFV MDVISRRDQRGSGQVVTYALNT I TNL KVOL I YOKV MD I I YRQEHRGSGQVVTYAFNT I TNMKVOL I CFAV MDVVGRRDQRGSGQVVTYALNT I TNGKVQVA TABV DLLIL I GKGQRCSGTVVTYSMNT I TNTVVOMM 595 621
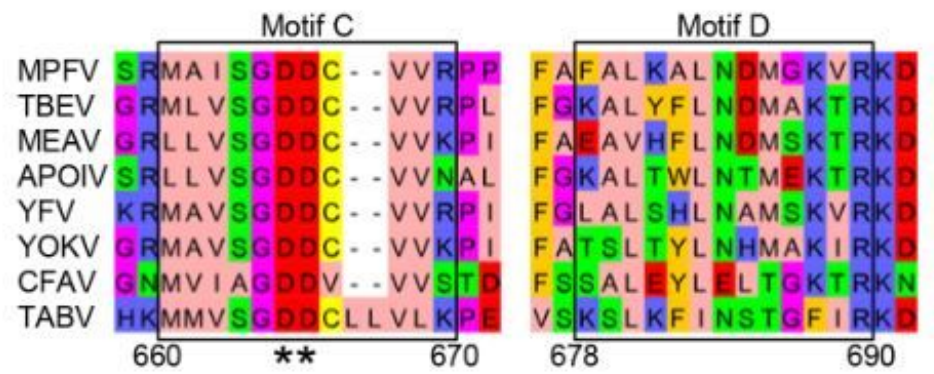

\section{Figure 1}

Conserved enzymatic motif in the serine protease, helicase/NTPase, methyltransferase, and RdRp. Sequence alignments of NS3 (a) and NS5 (b) from MPFV and representative flaviviruses were constructed. Enzymatic motifs are delimited accordingly. (a) The N-terminus and the C-terminus of NS3 contain the serine protease and helicase/NTPase, respectively. The catalytic triad of serine protease (Box 1-3) and seven conserved motifs of helicase/NTPase (Motifs I-VI) are highlighted. (b) The N-terminus and the C-terminus of NS5 contain 
methyltransferase and RdRp, respectively. The two (1 and 2) and four (A-D) conserved motifs of methyltransferase and RdRp are highlighted. Asterisks indicate conserved aspartic acid residues, which are important for enzymatic activity. Numbers at the bottom of the alignments refer to the MPFV sequence. Abbreviations of virus names are as follows: MPFV, Mpulungu flavivirus; TBEV, tick-borne encephalitis virus; MEAV, Meaban virus; APOIV, Apoi virus; YFV, Yellow fever virus; YOKV, Yokose virus; CFAV, Cell fusing agent virus; TABV, Tamana bat virus.

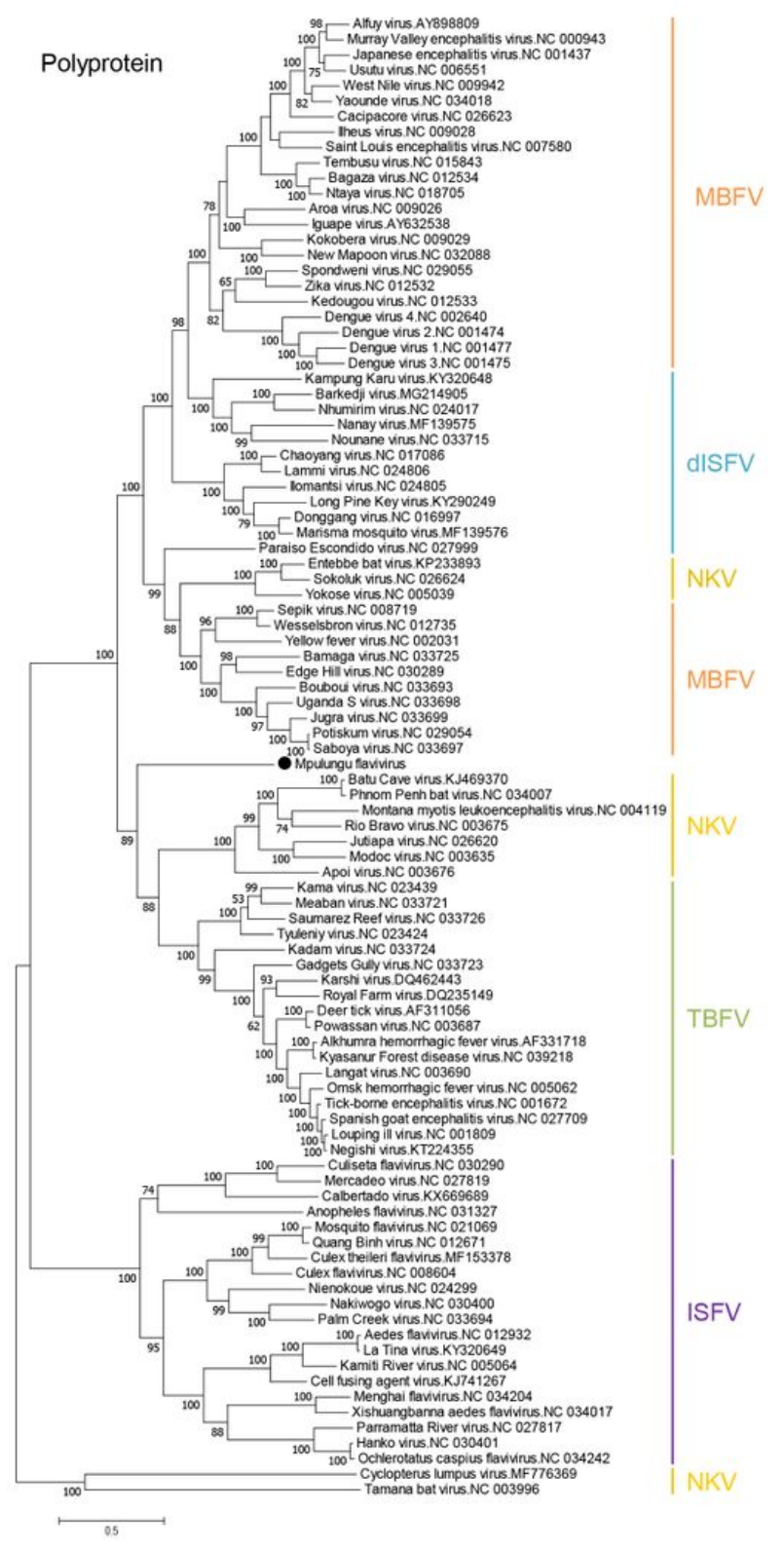

Fig. 2

\section{Figure 2}

Phylogenetic analyses of flaviviruses. A phylogenetic tree based on amino acid sequences of the polyprotein was constructed using the maximum likelihood method with 1,000 bootstrap replications. Bootstrap values $>50 \%$ based on 1,000 replications are shown on the interior branch nodes. Scale bar, 0.5 substitutions per site. Group names are indicated on the tree with abbreviations as follows: TBFV, tick-borne flavivirus; MBFV, mosquito-borne flavivirus; ISFV, 
insect-specific flavivirus; dISFV, dual-host affiliated insect-specific flavivirus; NKV, no known vector. Black circle represents MPFV

(a)

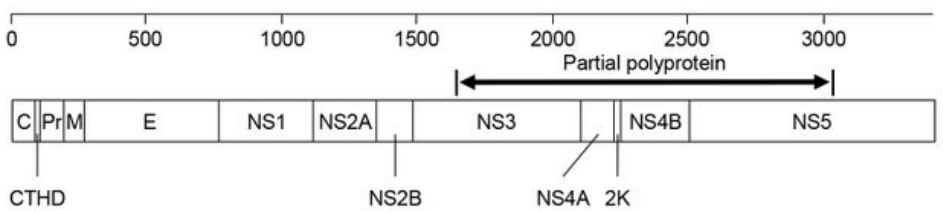

(b)

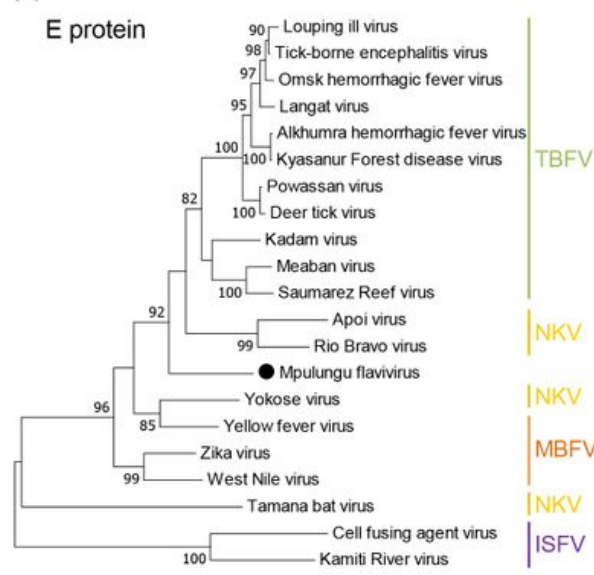
$\longmapsto 0.5$

(d)

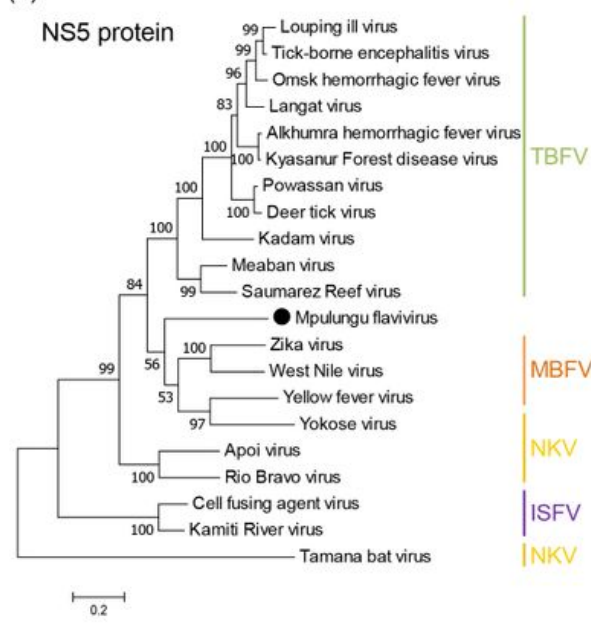

(c)

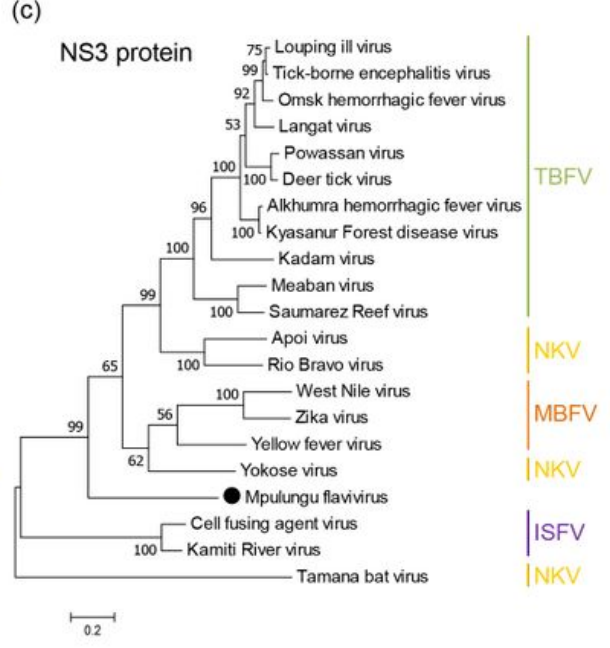

(e)

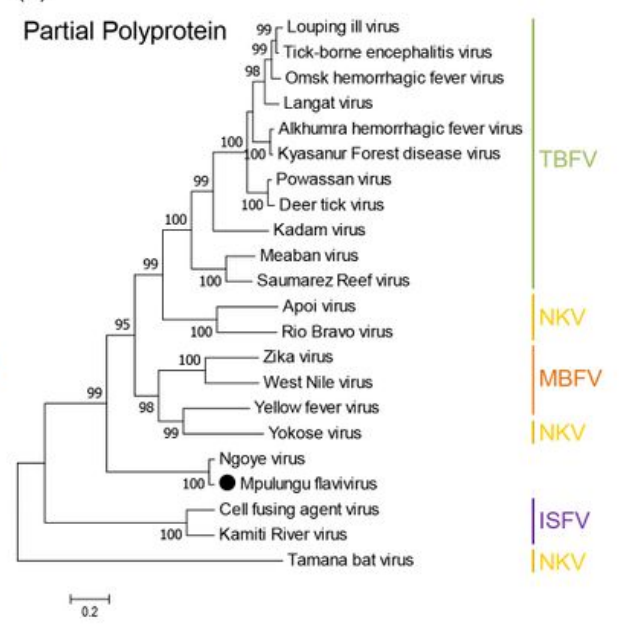

Fig. 3

\section{Figure 3}

Phylogenetic analyses of E, NS3, NS5 and partial polyprotein. (a) Schematic diagram of MPFV polyprotein. A black double-headed arrow represents the sequenced region of Ngoye virus. Trees were constructed using the maximumlikelihood method with 1,000 bootstrap replications as based upon 427-509 amino acid sequences for E (b), 577623 amino acid sequences for NS3 (c), 855-906 amino acid sequences for NS5 (d), and 1,392 amino acid sequences from the polyprotein corresponding to positions 1,642-3,033 of MPFV polyprotein (e). Bootstrap values > $50 \%$ based on 1,000 replications are shown on the interior branch nodes, and the scale bar indicates the number of substitutions per site. Group names are indicated on the trees. 
(a)

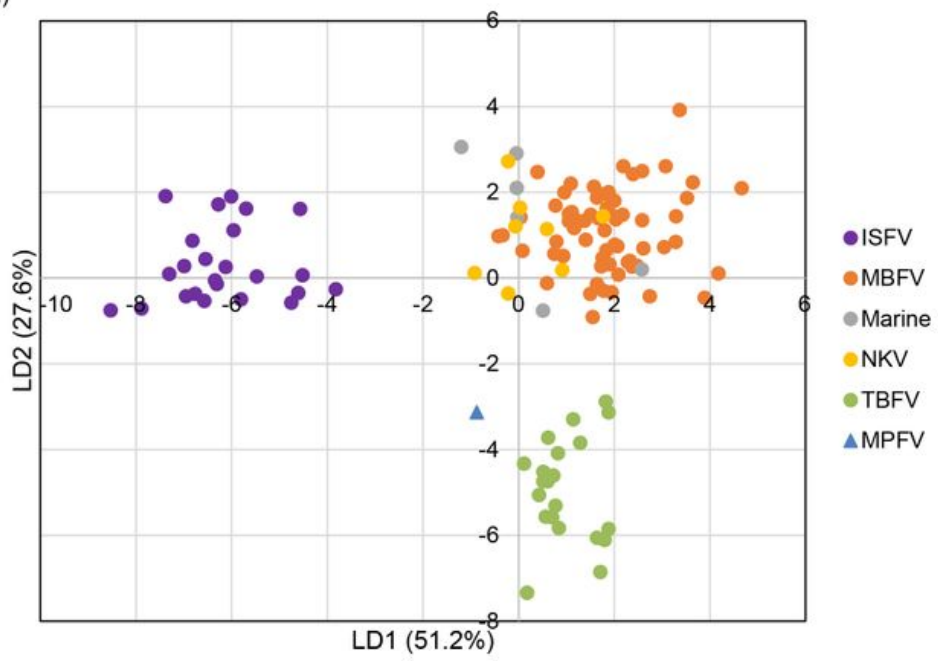

(b)

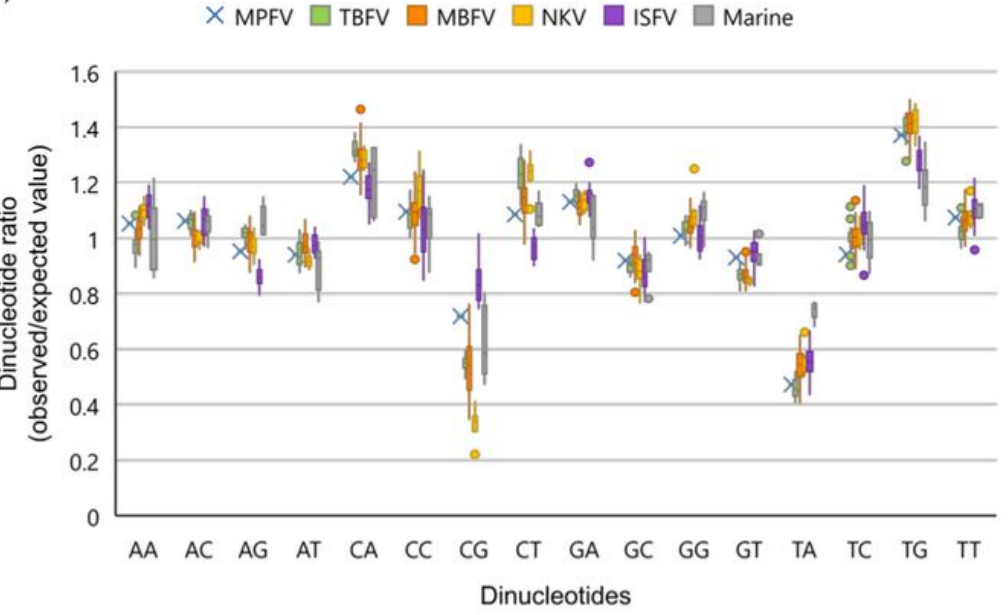

Fig. 4

\section{Figure 4}

Nucleotide composition analysis of flaviviruses. (a) Score plot of the linear discriminant analysis (LDA). The figure shows a scatterplot of the two discriminant scores explaining the largest amount of the components from LDA (51.2\% and $27.6 \%$ for LD1 and LD2, respectively). (b) Boxplot of dinucleotide ratios in flavivirus groups. Blue triangle (a) and blue cross (b) represent MPFV. Green: TBFV, orange: MBFV, yellow: NKV, purple: ISFV, gray: Marine. 
(a)

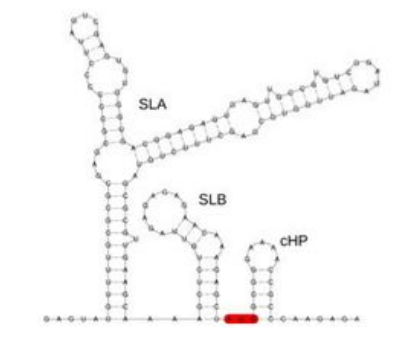

(b)

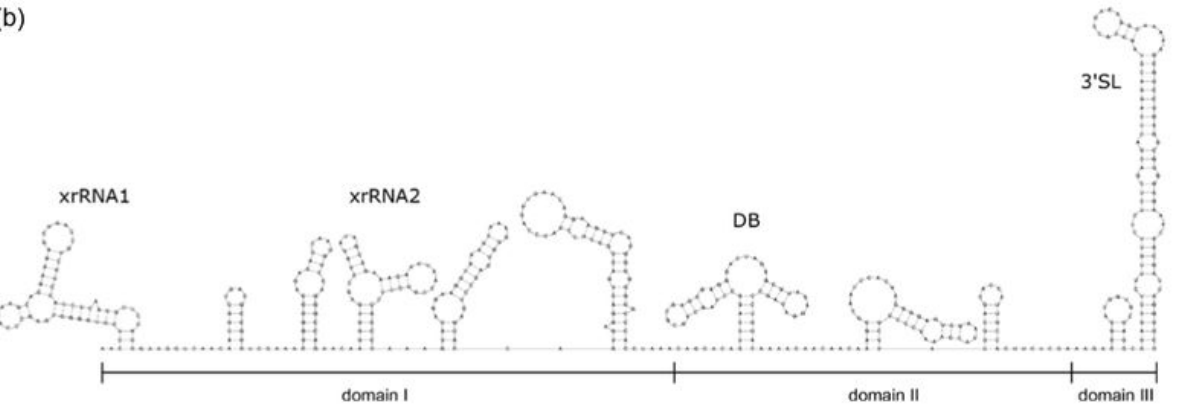

Fig. 5

\section{Figure 5}

Predicted secondary structures of the 5'- and the 3'-UTRs of MPFV. (a) Secondary structure prediction of the 5'terminus was performed using the nucleotide sequence of the $5^{\prime}$-UTR and the adjacent portion of the capsid protein. The canonical start codon is highlighted in red. The 5'-UTR of MPFV contains evolutionarily conserved elements, namely stem-loops A (SLA) and B (SLB), followed by the conserved capsid hairpin (cHP) structure at the beginning of the coding regions. (b) Secondary structure prediction of the 3'-UTR shows the overall architecture of this regulatory region, containing two exoribonuclease-resistant RNAs (xrRNA1 and xrRNA2), a dumbbell (DB) element and a long terminal 3 '-stem-loop (3'SL) structure. For the other stem-loop structures, no evolutionary support among other flaviviruses was obtained. 

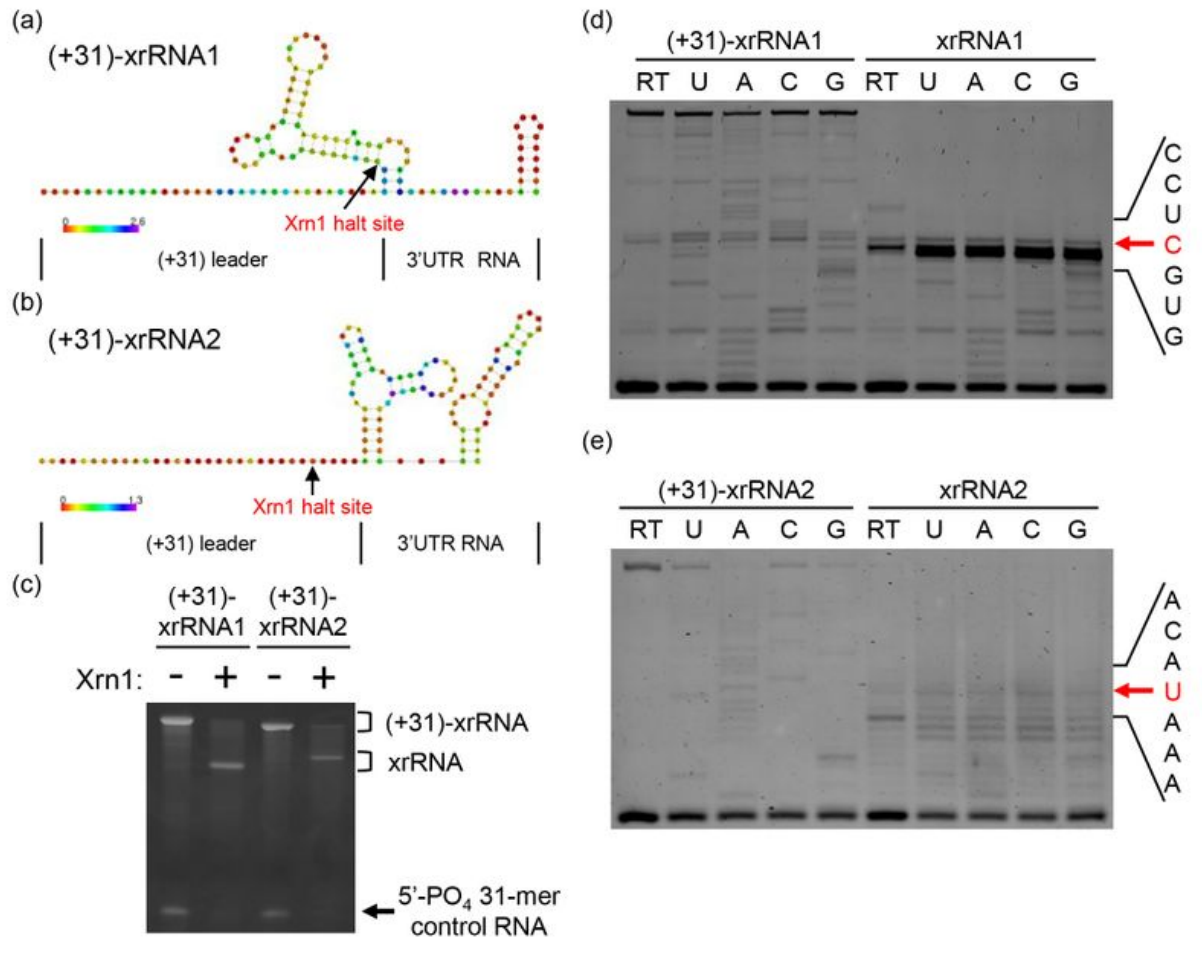

(d) $\frac{(+31)-x r R N A 1}{\text { RT U A C G RT U A C }} \frac{\text { GrRNA1 }}{\text { RT }}$

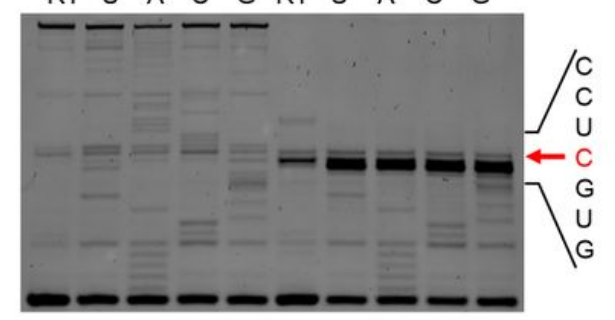

(e)

Fig. 6

\section{Figure 6}

Xrn1 degradation assay and characterization of the Xrn1-resistant products. $(a, b)$ Schematic diagram of predicted secondary structures of two RNA constructs, (+31)-xrRNA1 (a) and (+31)-xrRNA2 (b), used for the in vitro Xrn1 degradation assay. The leader sequences in these constructs are artificially designed 31-mers which do not form a prominent structure nor interact with the downstream genomic xrRNA sequences. Heat scale bars represent bits of positional entropy. Black arrows indicate the Xrn1 halt sites. (c) In vitro Xrn1 degradation assay using (+31)-xrRNA1 and (+31)-xrRNA2. This image is a part of full-length gel represented in Supplementary Fig. 3 (a). (d, e) Reverse transcription mapping the Xrn1 halt sites with RNA from panel (c). The location of the stop site (the 5 "border of the RNA products) is shown with a red arrow to the right, along with the sequence of the RNA surrounding this position. These images are parts of full-length gels represented in Supplementary Figs. 3 (b, c). 


\section{Supplementary Files}

This is a list of supplementary files associated with this preprint. Click to download.

- 2020Nov21HarimaHetal.Supplementalfiles.pdf 